\title{
iPSC-derived $\beta$ cells model diabetes due to glucokinase deficiency
}

\author{
Haiqing Hua, ${ }^{1,2}$ Linshan Shang, ${ }^{1}$ Hector Martinez, ${ }^{1}$ Matthew Freeby, ${ }^{2}$ Mary Pat Gallagher, ${ }^{2}$ \\ Thomas Ludwig, ${ }^{2}$ Liyong Deng, ${ }^{2}$ Ellen Greenberg, ${ }^{2}$ Charles LeDuc, ${ }^{2}$ Wendy K. Chung, ${ }^{2}$ \\ Robin Goland, ${ }^{2}$ Rudolph L. Leibel, ${ }^{2}$ and Dieter Egli'
}

${ }^{1}$ The New York Stem Cell Foundation Laboratory, New York, New York, USA. ${ }^{2}$ Division of Molecular Genetics, Department of Pediatrics and Naomi Berrie Diabetes Center, Columbia University, New York, New York, USA.

\begin{abstract}
Diabetes is a disorder characterized by loss of $\beta$ cell mass and/or $\beta$ cell function, leading to deficiency of insulin relative to metabolic need. To determine whether stem cell-derived $\beta$ cells recapitulate molecular-physiological phenotypes of a diabetic subject, we generated induced pluripotent stem cells (iPSCs) from subjects with maturity-onset diabetes of the young type 2 (MODY2), which is characterized by heterozygous loss of function of the gene encoding glucokinase (GCK). These stem cells differentiated into $\beta$ cells with efficiency comparable to that of controls and expressed markers of mature $\beta$ cells, including urocortin-3 and zinc transporter 8, upon transplantation into mice. While insulin secretion in response to arginine or other secretagogues was identical to that in cells from healthy controls, GCK mutant $\beta$ cells required higher glucose levels to stimulate insulin secretion. Importantly, this glucose-specific phenotype was fully reverted upon gene sequence correction by homologous recombination. Our results demonstrate that iPSC-derived $\beta$ cells reflect $\beta$ cell-autonomous phenotypes of MODY2 subjects, providing a platform for mechanistic analysis of specific genotypes on $\beta$ cell function.
\end{abstract}

\section{Introduction}

Recent progress in somatic cell reprogramming has allowed the generation of induced pluripotent stem cells (iPSCs) from diabetic subjects (1). Human pluripotent stem cells, including iPSCs and human ES cells, have the capacity to differentiate into insulin-producing cells (2), which display key properties of true $\beta$ cells, including glucose-stimulated insulin secretion upon maturation in vivo (3). iPSCs have been generated from patients with various types of diabetes $(2,4,5)$. However, whether iPSC-derived $\beta$ cells can accurately replicate pathologic phenotypes, and be used to test strategies to restore normal $\beta$ cell function, is not clear. As proof of principle, we chose to model a monogenic form of diabetes, maturity-onset diabetes of the young type 2 (MODY2) (6).

MODY is caused by single gene mutations, resulting in defects in the development, proliferation/regeneration, and/or function of $\beta$ cells (7). MODY accounts for 1 to 5 percent of all instances of diabetes in the United States (8), and MODY2, caused by mutations in the glucokinase (GCK) gene, accounts for $8 \%$ to $60 \%$ of all MODY cases, depending on population sampling $(9,10)$. GCK links blood glucose levels to insulin secretion by converting glucose to glucose-6-phosphate, the rate-limiting step in glycolysis. The catalytic capacity of GCK in $\beta$ cells determines the threshold for glucose-stimulated insulin secretion. Due to hypofunction of one allele of GCK, the dose-response curve relating glucose and insulin secretion obtained with graded glucose infusions is shifted to the right in the MODY2 subjects, resulting in mild hyperglycemia (11). Subjects with permanent neonatal diabetes, caused by the absence of both GCK alleles, are insulin dependent at birth and show intrauterine growth retardation (12). In a mouse model, heterozygous loss of GCK causes hyperglycemia, early-onset diabetes (10 weeks old), reduced response to glucose stimulation (13), and an inability to increase $\beta$ cell mass under conditions of insulin

Conflict of interest: The authors have declared that no conflict of interest exists. Citation for this article: J Clin Invest. 2013;123(7):3146-3153. doi:10.1172/JCI67638. resistance (14). Mouse islets with homozygous loss of GCK fail to increase insulin release in response to glucose in vitro (13).

These well-characterized consequences in mice and humans allow assessment of the accuracy of stem cell models for diabetes. Such models will offer significant advantages over a genetically manipulated mouse or human subjects for preclinical testing of therapeutic strategies and for drug screening as well as for studies designed to gain insight into the molecular mechanisms of how specific genotypes affect $\beta$ cell function and cause diabetes in human subjects. For example, while it is known that GCK affects glucose-stimulated insulin secretion, whether insulin biosynthesis and/or $\beta$ cell proliferation is also affected could not be determined in human subjects.

We found that iPSCs from MODY2 subjects heterozygous for hypomorphic GCK mutations differentiated into insulin-producing $\beta$ cells with an efficiency comparable to that of controls. In contrast, stem cells with 2 inactive GCK alleles showed a reduced capacity to generate insulin-producing cells. Hypomorphic GCK alleles reduced insulin secretion specifically in response to glucose but not in response to other secretagogues, including arginine. The responsiveness to glucose was restored when the GCK mutation was corrected by homologous recombination. These results demonstrate that iPSC-derived patient-specific $\beta$ cells recapitulate the anticipated functional phenotypes observed in human subjects and enable analysis of aspects of cellular physiology not otherwise possible.

\section{Results}

Stem cells with an allelic series at the GCK locus. We obtained skin biopsies from 2 MODY2 subjects: a 38-year-old woman of European descent diagnosed with diabetes at the age of 21 years and a 56-yearold man of European descent who was diagnosed with diabetes at the age of 47 years. Both of them had a family history of diabetes, were negative for antibodies associated with type 1 diabetes, nonobese $\left(\mathrm{BMI}=21-26 \mathrm{~kg} / \mathrm{m}^{2}\right)$, and positive for measurable, but low, 
A
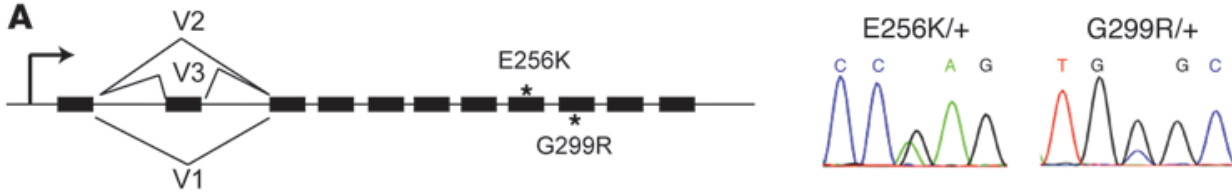

B
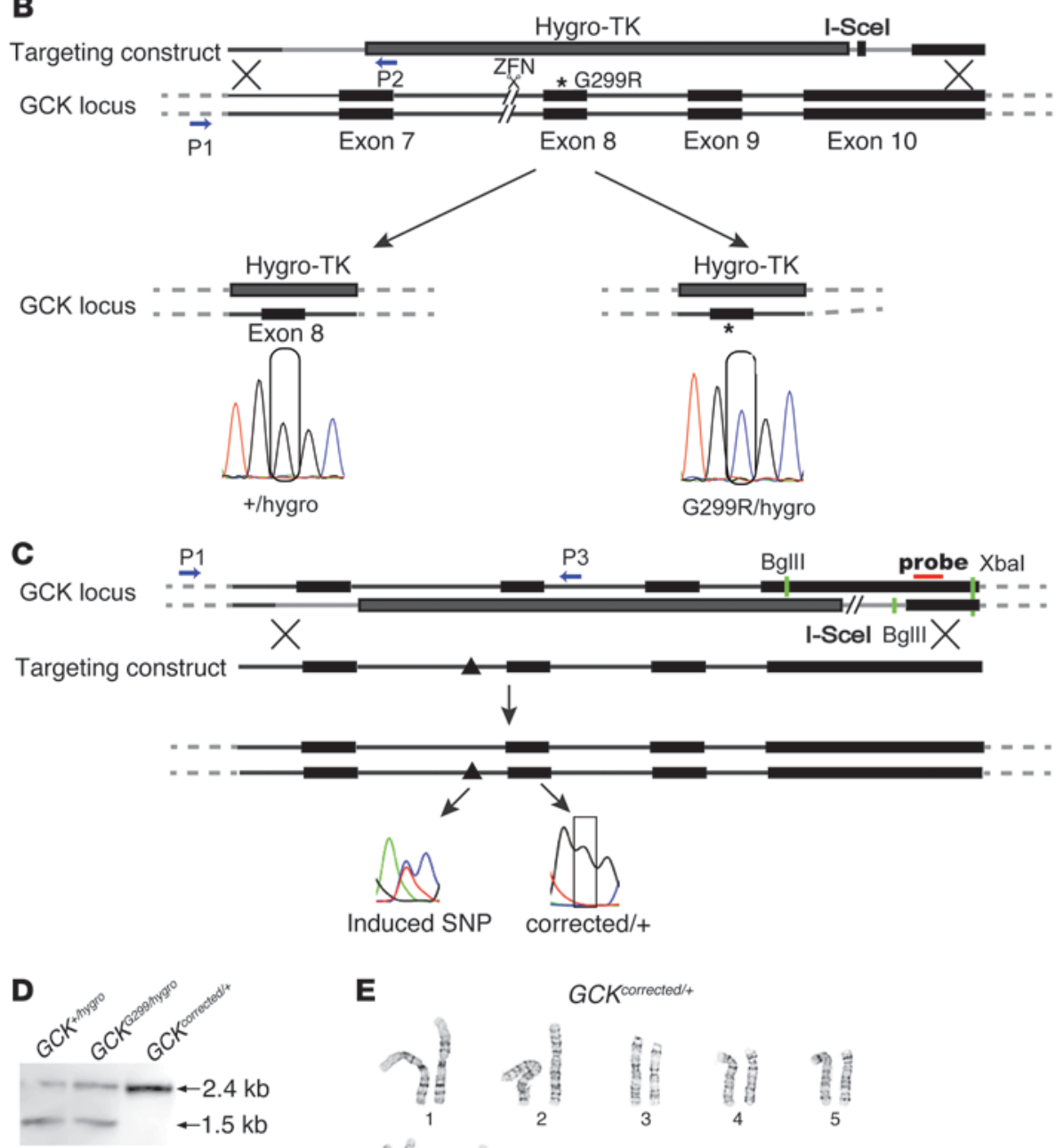

$\mathbf{E}$

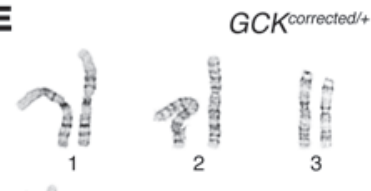

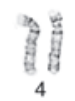
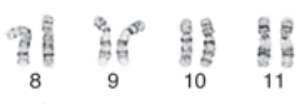
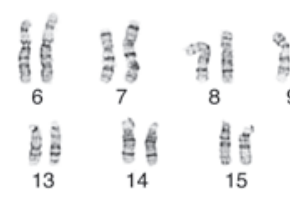

19

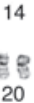

$+8$
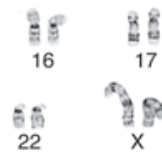

serum C-peptide (0.1-0.4 ng/ml) (Supplemental Figure 1; supplemental material available online with this article; doi:10.1172/ JCI67638DS1). MODY2 subjects typically display mild fasting hyperglycemia and can generally be managed with dietary therapy alone, while additional pharmacotherapy is sometimes used to optimally control blood glucose excursions (15). In the 2 MODY2 subjects from whom skin biopsies were obtained, diabetes control was excellent $(\mathrm{HbA} 1 \mathrm{C} \leq 6.5 \%)$ on insulin or sulfonylurea-related agents (Supplemental Table 1). Exonic sequencing of GCK revealed that the female subject carries a missense mutation (G299R) and the male subject carries a missense mutation (E256K) (Figure 1A).

Both mutations have been shown to be functionally hypomorphic, with less than $1 \%$ of activity of the wild-type allele (16).

iPSC lines were generated from skin cell lines using nonintegrating Sendai viruses (Supplemental Figure 2, A and B, and ref. 17). The iPSCs with the hypomorphic GCK mutations had the expression profile of pluripotent cells and the capability to differentiate into endodermal, mesodermal, and ectodermal tissues (Supplemental Figure 2, C-E). Because of genetic diversity in humans, controlling for effects of the genetic background is critical for functional comparisons between mutant and nonmutant cells (18). To generate cell lines with identical genetic background but with dif- 
A
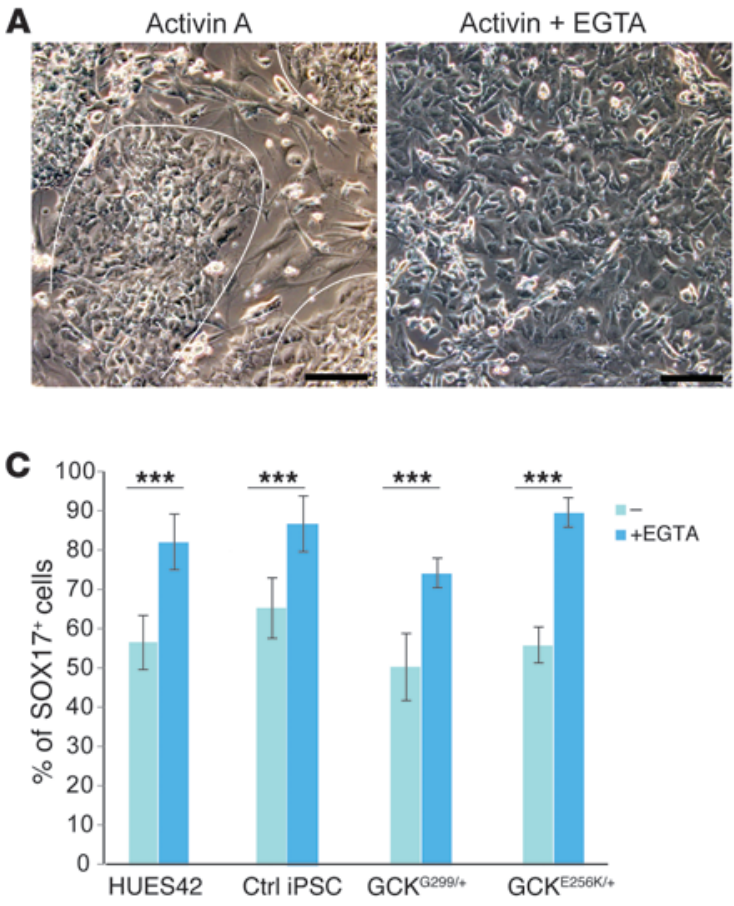

E

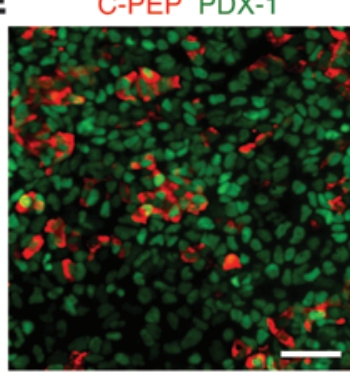

C-PEP NKX6.1

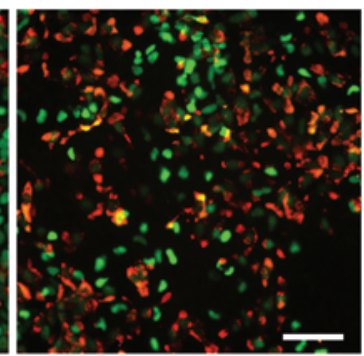

B

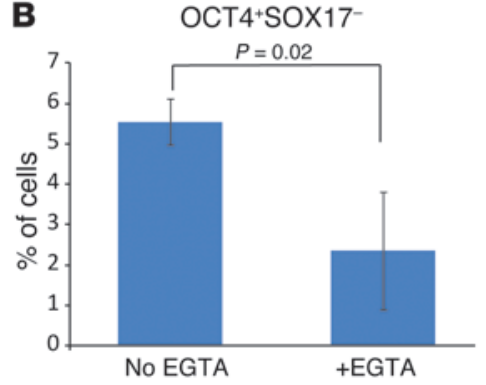

D

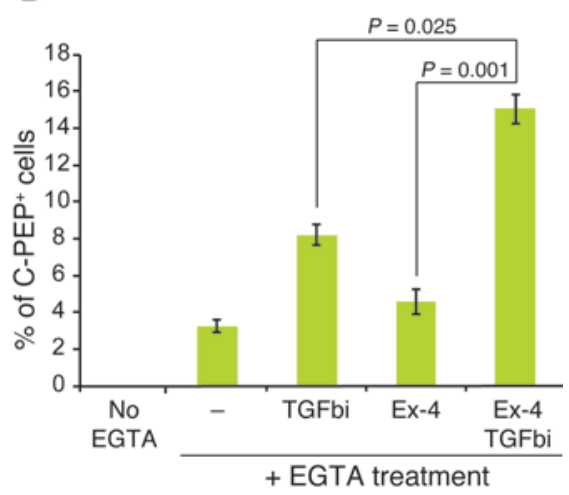

$\mathbf{F}$

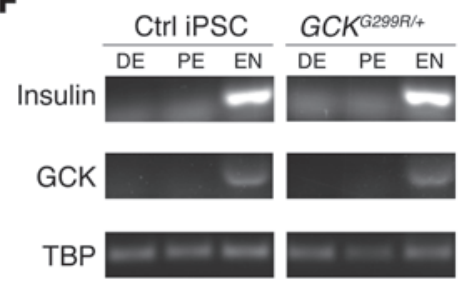

Figure 2

Enhanced $\beta$ cell generation through calcium chelation and TGF- $\beta$ signaling inhibition. (A) Morphology of control iPSCs after 1 day of activin A treatment with and without EGTA. Boundaries of colonies are indicated by white lines. Scale bar: $50 \mu \mathrm{m}$. (B and C) Differentiation of control iPSCs in the presence or absence of EGTA. Quantification of (B) OCT4positive SOX17-negative cells and (C) SOX17-positive cells after 3 days of differentiating control iPSCs. ${ }^{* \star} P<0.001$. (D) Percentage of insulin-positive cells (stained for C-peptide [C-PEP]) after treatment for 2 days with the indicated compounds ( $n=8$ replicas). (E) Immunostaining of $\beta$ cells derived in vitro (day 14). Scale bar: $50 \mu \mathrm{m}$. (F) The mRNA expression of INS and GCK at definitive endoderm (DE), pancreatic endoderm (PE), and endocrine (EN) stages of differentiation, determined by semiquantitative RT-PCR. TBP, TATA box binding protein. All error bars in this figure represent SEM. ferent genotypes at the GCK locus, we performed targeted genetic modifications (Figure 1B). We designed a 2-step targeting protocol that allowed the precise correction of the mutant base pair, without leaving a footprint of exogenous DNA. We first targeted the GCK locus with a linearized construct containing a PGK-hygro-TK fusion gene flanked by 2 segments of the GCK locus corresponding to intron 6 and exon 10 in $\mathrm{GCK}^{\mathrm{G} 299 R /{ }^{+}}$cells. mRNA encoding a zincfinger nuclease (ZFN) to induce a double-strand break 1,150 bp upstream of the G299R mutation was introduced into $G C K^{G 299 R /+}$ cells with the targeting plasmid to facilitate homologous recombination. Hygromycin-resistant colonies of transfected GCK $G^{G 299 R /+}$ cells were expanded and tested for homologous integration using PCR primers annealing to the genomic sequence and to the hygroTK cassette (Figure 1B). Of 201 hygromycin-resistant colonies, 14 (7\%) showed targeting of the construct to either the wild-type or the mutant allele, resulting in GCK ${ }^{G 299 R / \text { hygro }}$ and $G C K^{+/ \text {bygro }}$ cells, respectively (Figure 1C). Cells carrying the GCK ${ }^{\text {hygro }}$ allele with exons 7-10 disrupted, in combination with the G299R mutation, are expected to have very little, if any, GCK activity.

In a second step, 2 wild-type copies of GCK were restored in $G C K^{+/ h y g r o}$ cells using a plasmid containing the wild-type GCK locus, which was marked with a SNP in intron 7 to distinguish the 2 copies of GCK. A plasmid encoding the endonuclease I-SceI site was cotransfected to induce a double-strand break at an I-SceI recognition site located in the hygro-TK cassette to facilitate homologous recombination and to replace all vector sequences, including the TK gene (Figure 1B). Ganciclovir-resistant colonies were screened for homologous integration using PCR with one primer outside of the targeting construct and one primer within the construct, followed by sequencing of the induced SNP. Two out of 96 colonies ( $2 \%$ efficiency) had correctly targeted to the GCK locus and restored 2 wild-type copies of GCK, and this was also confirmed by Southern blotting (Figure 1D); these cells were karyotypically normal (Figure $1 \mathrm{E}$ ) and designated $\mathrm{GCK}^{\text {corrected/+}}$. These targeted manipulations resulted in an allelic series of cells that were wild-type $\left(G C K^{\text {corrected/+ }}\right)$, hypomorphic $\left(G C K^{G 299 R /+}\right)$, and/ or null $\left(G C K^{\mathrm{G} 299 R / h_{y g r o}}\right)$ for GCK function on the same genetic background, allowing us to exclude potential confounding effects of different genetic backgrounds in subsequent experiments.

Efficient $\beta$ cell generation from GCK-deficient iPSCs. Human ES cells and iPSCs can be differentiated toward insulin-producing cells after stepwise differentiation into definitive endoderm $\left(\mathrm{SOX} 17^{+}\right)$, pan- 
creatic progenitors $\left(\mathrm{PDX}^{+}\right)$, and endocrine progenitors $\left(\mathrm{NGN}^{+}\right)$ $(2,19)$. While published protocols yielded SOX17- and PDX1-positive cells, insulin-producing cells were not obtained (Supplemental Figure 3A). We noticed that 3 days after induction of differentiation (stage 1), colonies with the morphology of pluripotent stem cells were still apparent. These cells retained OCT4 expression and failed to commit to the endoderm lineage, as evidenced by the lack of SOX17 expression (Supplemental Figure 3B). We reasoned that interfering with the pluripotent state should increase the ability of activin to direct differentiation toward the endoderm lineage. Cellto-cell interactions mediated by E-cadherin are critical for maintaining pluripotency of ES cells (20). When the calcium chelator EGTA, an inhibitor of cadherin-mediated cell-cell attachment, was added on the first day of differentiation, the tight colony morphology of iPSCs was lost (Figure 2A). In parallel, the percentage of OCT $4^{+} \mathrm{SOX} 17^{-}$cells was reduced from $5 \%$ to $2 \%$ (Figure $2 \mathrm{~B}$ ), while the percentage of endodermal (SOX $17^{+} \mathrm{OCT}^{-}$) cells was increased by $25.5 \%$ (Figure $2 \mathrm{C}$ ). These responses, in the aggregate, resulted in a $21.7 \%$ (mean of 4 different cell lines) increase in PDX1-positive cells on day 8 of differentiation (stage 3) (Supplemental Figure $3 \mathrm{C}$ ). To further improve differentiation conditions from pancreatic progenitor to $\beta$ cells, exendin-4, a glucagon-like peptide- 1 agonist, and SB431542, a TGF- $\beta$ signaling inhibitor, were added to stage 3 progenitor cells. Both of these additions enhanced the differentiation efficiency of $\beta$ cells to $4.6 \%$ and $8.2 \%$ (C-peptide ${ }^{+}$), respectively, consistent with previous observations (21-23). A combination of exendin-4 and SB431542 treatment from day 9 to day 12 produced the highest percentage of $\beta$ cells (15\%) (Figure 2D). We found that our modified protocol efficiently induced differentiation of both ES cells and iPSCs (Figure 2D and below and Supplemental Figure $3 \mathrm{D})$. When differentiated into $\beta$ cells, control and MODY2 stem cells showed similar efficiency of generating C-peptide ${ }^{+}$cells from $\mathrm{PDX} 1^{+}$progenitors (Supplemental Figure 3D). These cells expressed $\beta$ cell transcriptional factor PDX1 and NKX6.1 (Figure $2 \mathrm{E})$. To assess the temporal expression pattern of GCK during the in vitro differentiation process, we measured GCK mRNA levels at definitive endoderm (day 3 of differentiation), pancreatic endoderm (day 8), and endocrine (day 12) stages. Expression of GCK was detected only at the endocrine stage, coinciding with the expression of insulin (Figure 2F). We observed that in our differentiation culture $38 \%$ of the insulin-producing cells also immunostained for glucagon and $14 \%$ of the insulin-producing cells also expressed somatostatin, similar to previous observations (ref. 19 and Supplemental Figure 3E). Cells coproducing insulin and glucagon also appear during development of human fetal pancreas (24), suggesting that these cells were not fully differentiated. Further differentiation into monohormonal cells occurred in vivo, after transplantation of cells at day 12 of differentiation under the kidney capsule of immune-compromised mice. Three months after transplantation, 24 out of 50 mice had detectable human C-peptide in their sera (Figure 3A). To determine whether the C-peptide originated from the transplants, we removed the transplants from 7 mice and found that none retained detectable human C-peptide in the sera (Figure 3B). Immunohistochemistry of the isolated graft showed that hormone-expressing cells in the transplants expressed solely insulin, glucagon, or somatostatin (Figure 3C). We also observed the presence of urocortin- 3 and zinc transporter 8 in the insulinpositive cells in the transplants (Figure 3D), while these markers of mature $\beta$ cells were absent in $\beta$ cells derived in vitro (Supplemental Figure 4A and refs. 25, 26).
GCK mutations specifically affect glucose-mediated insulin secretion. $\beta$ Cells in MODY2 patients with GCK mutations are able to respond to glucose but with reduced sensitivity (11). To determine whether this phenotype can be recapitulated by iPSCderived $\beta$ cells, we performed intraperitoneal glucose tolerance tests on transplanted mice. We measured both human C-peptide and glucose concentrations in the blood and found a dose responsiveness of $\mathrm{C}$-peptide to circulating blood glucose concentration. We evaluated the sensitivity of human insulin-producing transplanted cells by assessing the slopes of these relationships. $\mathrm{GCK}^{\mathrm{G} 299 R /+}$ cells showed a reduced sensitivity to glucose compared with control cells (Figure 3E). Gene correction in GCK ${ }^{\text {corrected/+ }}$ cells restored glucose sensitivity to that of control cells. If GCK effects are mediated solely by impact on glucose sensing, insulin secretion in response to secretagogues acting independently of glycolysis should be unaffected. To test this possibility, we first performed glucose-stimulated insulin secretion assays on in vitro differentiated $\beta$ cells. In order to bracket physiologically relevant concentrations of glucose, we treated iPSC-derived $\beta$ cells and human islets with $5.6 \mathrm{mM}$ and $16.9 \mathrm{mM}$. $2.5 \mathrm{mM}$ and $20 \mathrm{mM}$ glucose were also used to treat control and MODY2 iPSC-derived $\beta$ cells. $\beta$ Cells derived from human ES cells and control iPSCs showed increased C-peptide secretion (mean: 2.1 fold; range $0.8-3.5 ; 21$ out of 28 biological replicates showed $>1.2$-fold increase). In contrast, $G C K^{E 256 K /+}, G C K^{G 299 R /+}$, and $G C K^{G 299 R / h y g r o ~}$ cells showed no increase (mean: 0.9 fold; range 0.7-1.1; none of the 25 biological replicas showed $>1.2$-fold increase) (Figure $4 \mathrm{~A}$ and Supplemental Figure 4B). Importantly, correction of the G299R mutation to the wild-type nucleotide sequence restored glucose responsiveness: GCK ${ }^{\text {corrected/+ }}$ cells showed a 1.6-fold increase in glucose-stimulated C-peptide secretion (range: 1.1-2.3; 4 out of 5 biological replicas showed $>1$.2-fold increase; $P=0.003$ ) (Figure 4A). When exposed to other secretagogues, GCK ${ }^{\mathrm{G} 299 R /+}$ and $G_{C K}^{\mathrm{G} 299 R / \text { bygro }}$ cells increased C-peptide release in response to arginine ( 3 to 4 fold), potassium ( 3 to 4 fold), and Bay K8644, a calcium channel agonist ( 3 to 5 fold), identical to control cells (Figure 4B and Supplemental Figure 4B). Therefore, GCK mutations specifically affect glucose-mediated insulin secretion.

GCK mutations may also affect other aspects of $\beta$ cell function, such as production/processing of insulin precursors or interference with insulin secretion or $\beta$ cell proliferation. These different possibilities have not been addressed in human cells thus far. We found that insulin content was comparable in control $\beta$ cells and cells with $G_{C K} K^{G 299 /++}, G C K^{G 299 R / b y g r o}$, and GCK ${ }^{\text {corrected/+ }}$ genotypes (Figure 4C). By electron microscopy, cellular granule morphology and numbers were comparable in wild-type cells (average 173 granules per cross-section) and CCK $^{G 299 R /+}$ cells (average 220 granules per cross-section) (Supplemental Figure 4, C and D). We also found that heterozygous loss of GCK didn't alter the yield of $\beta$ cells from $\mathrm{PDX} 1^{+}$progenitors, but a reduction in $\beta$ cell generation was observed in GCK ${ }^{\mathrm{G} 299 R / \text { hygro }}$ cells $(5 \%$ C-peptide positive in $G C K^{\mathrm{G} 299 R / h y g r o}$ cells versus $10 \% \mathrm{C}$-peptide positive in $\mathrm{GCK}^{\mathrm{G} 299 R /{ }^{+}}$or $\mathrm{GCK}^{\text {corrected/+ }}$ cells) (Figure $4 \mathrm{D}$ ). This difference could be caused by reduced replication of $\beta$ cells, because we observed a reduction of Ki67-positive $\beta$ cells (31\% of C-peptide-positive cells) in the $G C K^{G 299 R / h y g r o}$ genotype, compared with the $G K^{\mathrm{G} 299 R /+}(41 \%), G^{\text {corrected/+ }}(45 \%)$, and control $\mathrm{GCK}^{+/+}$(HUES42, 49\%) genotypes (Figure 4E). Therefore, haploinsufficiency of GCK does not affect insulin biosynthesis and proliferation of iPSC-derived $\beta$ cells in vitro. 

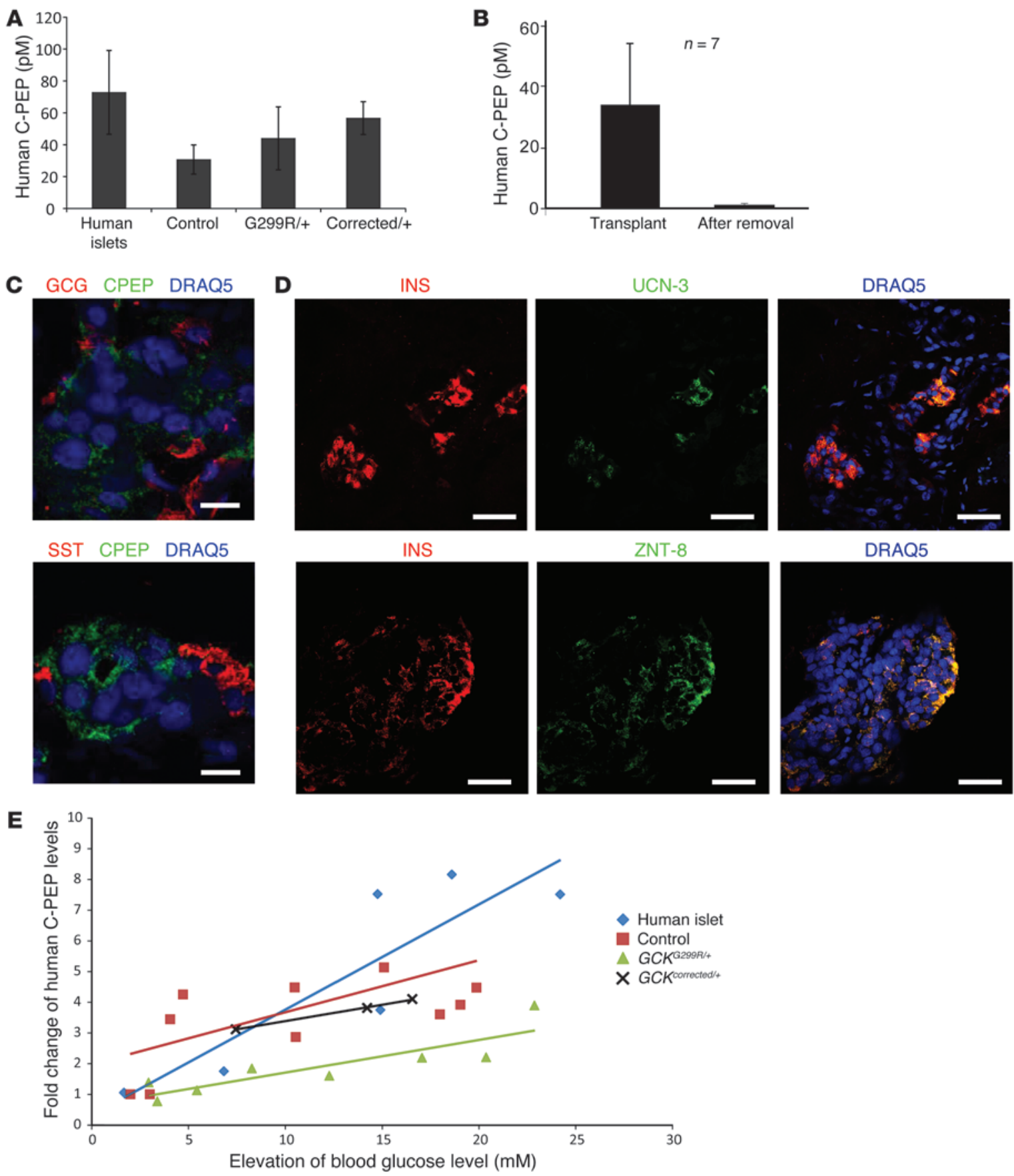

Figure 3

$\beta$ Cells derived in vivo display characteristics of mature $\beta$ cells. (A) Human C-peptide concentrations in mouse sera collected in the fasting state. Error bars represent standard deviation. (B) Measurement of human C-peptide levels in mouse sera prior to and after excision of the transplants. Mice transplanted with GCK mutant cells $\left(G_{C K}{ }^{G 299 R /+}\right)$ are shown. Error bars represent standard deviation. (C and $\left.\mathbf{D}\right)$ Immunohistochemistry of explants isolated 4 months after transplantation of GCK mutant cells (GCK ${ }^{G 299 R /+}$ ). GCG, glucagon; SST, somatostatin; INS, insulin; UCN-3, urocortin-3; ZNT8, zinc transporter 8. Scale bar: $10 \mu \mathrm{m}$ (C); $100 \mu \mathrm{m}$ (D). (E) Scatter plots showing fold change in C-peptide concentration (30 minutes after glucose injection versus 16 hours of fasting) versus the change in capillary blood glucose concentration (30 minutes after glucose injection minus 16 hours of fasting) during intraperitoneal glucose tolerance tests.

\section{Discussion}

In this study, we tested the fidelity with which $\beta$ cell-autonomous defects in a monogenic form of diabetes are reflected by iPSCderived insulin-producing cells. We found that MODY2 $\beta$ cells responded to elevated glucose with lower sensitivity compared with gene-corrected control cells but were otherwise comparable to control cells in insulin production and processing and insulin secretion in response to other secretagogues, such as arginine. These 
A

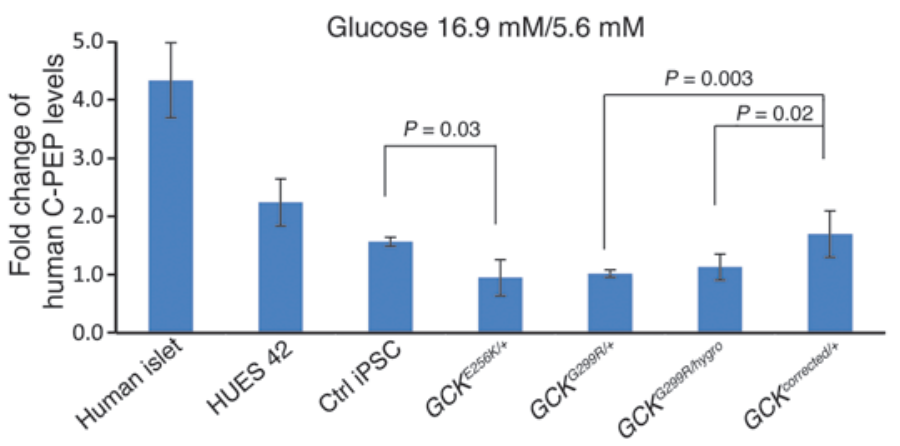

B

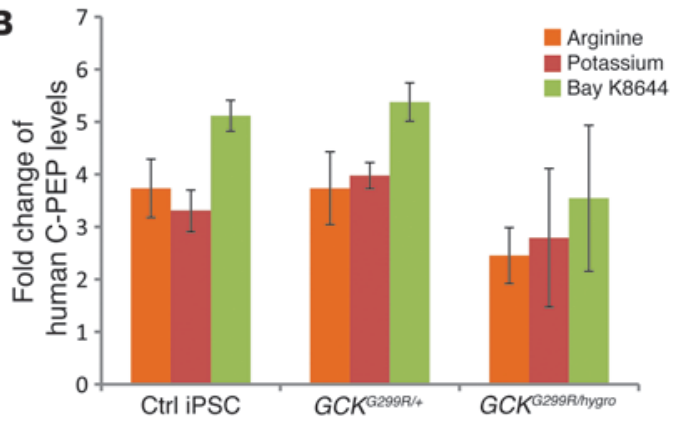

C

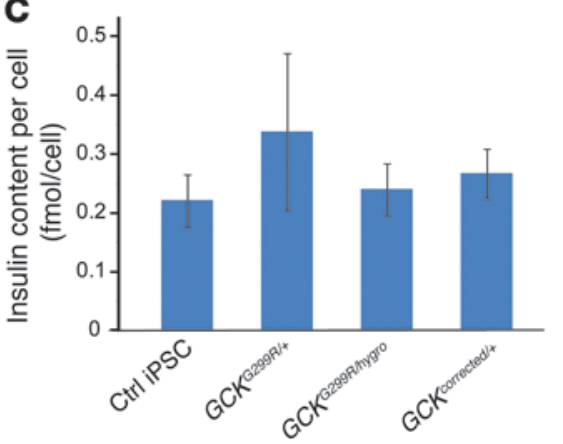

D

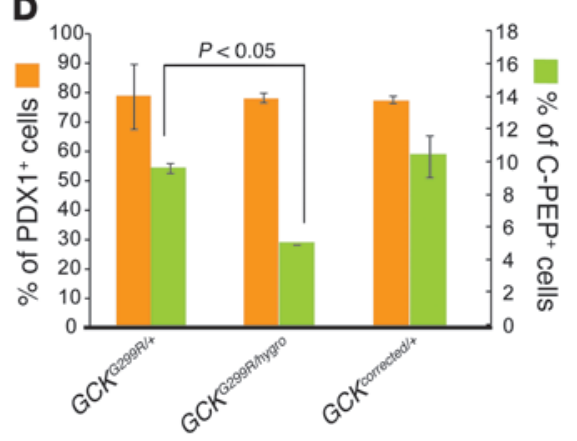

E

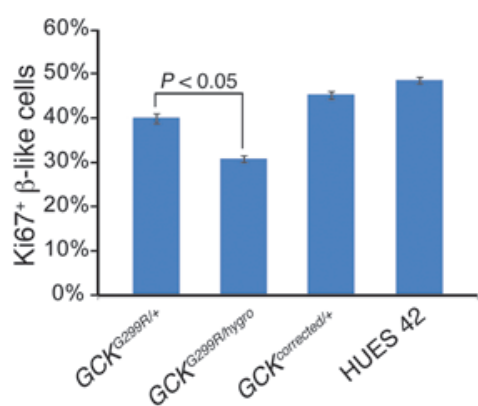

Figure 4

GCK gene dosage specifically affects glucose-stimulated insulin secretion. (A) Fold change of glucose-stimulated insulin (C-peptide) secretion in human islets and control, GCK mutant, and gene-corrected cells in vitro. The basal condition was $5.6 \mathrm{mM}$ glucose, and the stimulation condition was $16.9 \mathrm{mM}$ glucose. Error bars represent standard deviation of 3 experiments. (B) Insulin (C-peptide) secretion in response to indicated secretagogues in vitro. (C) Insulin content of control cells and GCK mutant and gene-corrected cells calculated with total insulin content and numbers of insulin-positive cells. (D) Differentiation efficiency of GCK G299R mutant and gene-corrected cells. (B-D) Error bars represent standard deviation. (E) Proportion of in vitro-differentiated $\beta$ cells (C-peptide positive) that were Ki67 positive. Error bars represent SEM ( $n=20$ replicates).

findings demonstrate that cells heterozygous for hypomorphic GCK mutations recapitulate key aspects of the MODY2 phenotype.

The observation of anticipated phenotypes using iPSC-derived $\beta$ cells suggests that differences between GCK mutant and control cells that cannot be readily investigated in human cells may also reflect aspects of the human disease. We found that in vitrodifferentiated $\beta$ cells carrying 2 inactive GCK alleles, but not cells with 1 or 2 functional GCK alleles, yielded a lower number of $\beta$ cells, at least partially as a result of effects on proliferation. Though the $\beta$ cells generated in vitro show high rates of proliferation that are more similar to those of the embryonic than the adult pancreas (27), GCK is expressed in $\beta$ cells of fetal islets (28, 29), and Porat et al. recently demonstrated a role for GCK in regulating $\beta$ cell proliferation in adult mice (30). We also found that in vitro, but not upon further differentiation in vivo, GCK mutant $\beta$ cells failed to increase insulin secretion at high ambient glucose concentrations. Whether this difference reflects an involvement of GCK in establishing responsiveness to glucose during functional maturation of $\beta$ cells remains to be investigated.

iPSC-derived cells should enable novel insights into the molecular cell biology of $\beta$ cell failure in virtually all forms of diabetes. Stem cell models of diabetes should not only allow deeper insight into the consequences of specific mutations on $\beta$ cell function but, in doing so, also shed light on the molecular physiology of the $\beta$ cell in prevalent clinical circumstances. Common variants of WFS1
(31), KCNJ11 (32), and GCK $(33,34)$ increase the risk of type 2 diabetes. Stem cell-based approaches may also allow the investigation of genes modifying penetrance of specific mutations that affect $\beta$ cell function. Importantly, we were also able to demonstrate that the specific correction of the mutant base pair in the GCK locus by homologous recombination restores glucose-stimulated insulin secretion. Our system of homologous recombination offers a significant advantage over previously reported techniques, as it is both efficient and does not result in the introduction of exogenous DNA sequences, such as loxP sites (35). The generation of autologous $\beta$ cells in combination with gene correction may ultimately be useful for cell replacement to restore normal glucose homeostasis.

\section{Methods}

Research subjects and cell lines. Biopsies of upper arm skin were obtained from 2 MODY2 subjects and a healthy subject using local anesthesia (lidocaine) and an Acu-Punch Biopsy Kit (Acuderm Inc.). Samples were coded and transported to the laboratory. Biopsies were cut in 10 to 12 small pieces, and 2-3 pieces of minced skin were placed around a silicon droplet in a well of a 6-well dish. A glass cover slip was placed over the biopsy pieces, and $5 \mathrm{ml}$ biopsy plating media was added. After 5 days, biopsy pieces were grown in culture medium for 3 to 4 weeks. Biopsy plating medium was composed of DMEM, FBS, GlutaMAX, Anti-Anti, NEAA, 2-Mercaptoethanol, and nucleosides (all from Invitrogen), and culture medium contained DMEM, FBS, GlutMAX, and Penicillin/Streptomycin (all from Invitrogen). 
HUES42 was chosen from a collection of Harvard University ES cell lines based on its robust and consistent ability to produce $\beta$ cells in vitro (36).

Generation of iPSCs. Primary fibroblasts were converted into pluripotent stem cells using the CytoTune-iPS Sendai Reprogramming Kit (Invitrogen). 50,000 fibroblast cells were seeded per well in a 6-well dish at passage 3 and allowed to recover overnight. The next day, Sendai viruses expressing human transcription factors OCT4, SOX2, Klf4, and C-Myc were mixed in fibroblast medium to infect fibroblast cells according to the manufacturer's instructions. Two days later, the medium was exchanged with human ES medium supplemented with the ALK5 inhibitor SB431542 (2 $\mu \mathrm{M}$; Stemgent), the MEK inhibitor PD0325901 (0.5 $\mu \mathrm{M}$; Stemgent), and thiazovivin $(0.5 \mu \mathrm{M}$; Stemgent). Human ES medium contained KODMEM, KSR, GlutMAX, NEAA, 2-Mercaptoethanol, Penicillin/Streptomycin, and bFGF (all from Invitrogen). On day 7-10 after infection, cells were detached using TrypLE and passaged onto feeder cells. Individual colonies of iPSCs were picked between days 21 and 28 after infection, and each iPSC line was expanded from a single colony. All iPSCs lines were cultured on mouse embryonic fibroblast cells with human ES medium. Karyotyping was performed by Cell Line Genetics Inc. For teratoma analysis, 1-2 million cells from each iPSC line were detached and collected after TrypLE (Invitrogen) treatment. Cells were suspended in $0.5 \mathrm{ml}$ human ES media. The cell suspension was mixed with $0.5 \mathrm{ml}$ Matrigel (BD Biosciences) and injected subcutaneously into dorsal flanks of an immunodeficient mouse (NOD.Cg-Prkd scid Il2 $r^{t m l W j l} /$ SzJ, stock no. 005557, The Jackson Laboratory) (37). Eight to twelve weeks after injection, teratomas were harvested, fixed overnight with $4 \%$ paraformaldehyde, and processed according to standard procedures for paraffin embedding. The samples were then sectioned and H\&E stained.

Gene expression analysis. Total RNA was isolated from cells with the RNAeasy Kit (Qiagen). For quantitative PCR analysis, cDNA was synthesized using the Promega RT system (Promega). Primers for qRT-PCR are listed in Supplemental Table 2. For microarray analysis, RNA was prepared using the Illumina Total Prep RNA Amplification Kit (Ambion) and hybridized to HumanRef-8 v3 BeadChip Kit (Illumina). The global expression profiles of the samples were analyzed, with normalization to average and subtraction of background using GenomeStudio Software (Illumina), and a hierarchical cluster tree was generated based on the correlation coefficients among samples. All array data are available at the Gene Expression Omnibus (accession no. GSE45777).

Directed differentiation into $\beta$ cells. ES cells or iPSCs were dissociated using Dispase (3-5 minutes) and, subsequently, Accutase (both from Invitrogen) (5 minutes). Cells were suspended in human ES medium containing $10 \mu \mathrm{M}$ ROCK inhibitor (Y27632) and filtered through a 70- $\mu \mathrm{m}$ cell strainer. Cells were then plated at a density of 400,000 cell per well in 12-well plates. After 1 or 2 days, when cells reached $80 \%-90 \%$ confluency, differentiation was started. Detailed formulations of differentiation media are listed in Supplemental Table 3. Typically, cells were assayed between day 12 and day 16. For measuring proliferation rate, cells were assayed at day 12. Insulin contents were measured using the Insulin ELISA Kit (Mercodia).

Gene targeting. A targeting vector (pBS-PGK-hytk-IsceI-LoxP) was constructed by cloning a PGK-hygro-TK cassette into a pBlueScript $\mathrm{SK}^{+}$vector. A LoxP site was added upstream of the cassette. A LoxP and an I-SceI site were cloned downstream of the cassette. Two DNA fragments, "homologous arms," from the GCK gene (see Supplemental Table 2 for primer sequences) were cloned into the $\mathrm{pBS}$-PGK-hytk-IsceI-LoxP vector at $5^{\prime}$ and $3^{\prime}$ end of the cassette. A correction construct was created by cloning a DNA fragment of GCK (see Supplemental Table 2 for primer sequences) into pCR2.1-TOPO vector using the TOPO TA Cloning Kit (Invitrogen).

A pair of ZFNs was designed by Sigma-Aldrich to recognize the following sequence in intron 7 of GCK: CGTCAATACCGAGTGgggcgcCTTC-
GGGGACTCCGGC (with uppercase letters representing the ZFNbinding site and lowercase letters representing the cut site). $5 \mu \mathrm{g}$ of each ZFN-encoding plasmid (RNA) and $5 \mu \mathrm{g}$ of the targeting plasmid (DNA digested with ClaI and NotI, gel purified) were used to transfect 1 million $\mathrm{GCK}^{\mathrm{G} 299 R /+}$ cells. Transfection was performed using Amaxa Nucleofector (program A-13) and Human Stem Cell Solution I (Lonza). After transfection, cells were seeded on a $10-\mathrm{cm}$ culture dish and allowed to recover for 2 days. Cells were then selected by 2 days of exposure to hygromycin (50 $\mu \mathrm{g} / \mathrm{ml})$. Resistant colonies were screened by PCR. The $G C K^{+/ \text {hygro }}$ cells were transfected by $5 \mu \mathrm{g}$ of the correction plasmid and $5 \mu \mathrm{g}$ of a plasmid carrying the I-SceI enzyme using the method described above. After transfection, cells were treated with $2 \mu \mathrm{g} / \mathrm{ml}$ ganciclovir for 2 days. PCR and sequencing were used to screen the resistant colonies, and Southern blotting was used to confirm targeted integration. Southern blotting was performed using the DIG System following the manufacturer's instruction (Roche). Primers for probe synthesis are listed in Supplemental Table 2. DNA from stem cells was prepared using the High Pure PCR Template Preparation Kit (Roche). $10 \mu \mathrm{g}$ of DNA from each cell line was digested with BglII and XbaI.

Immunostaining. Cultured cells were briefly washed with PBS and fixed with $4 \%$ paraformaldehyde for 30 minutes at room temperature. Embryoid bodies and mouse kidneys were fixed with $4 \%$ paraformaldehyde overnight at $4^{\circ} \mathrm{C}$, dehydrated using $15 \%(\mathrm{w} / \mathrm{v})$ sucrose and $30 \%(\mathrm{w} / \mathrm{v})$ sucrose solution, embedded in OCT compound (Tissue-Tek), and frozen at $-80^{\circ} \mathrm{C}$. Fixed cells or frozen sections were blocked in $5 \%$ normal donkey serum for 30 minutes. Primary antibodies used in the study were as follows: mouse anti-C-peptide (05-1109; Millipore), goat anti-glucagon (A056501; DAKO), goat anti-PDX1 (AF2419; R\&D Systems), goat antiSOX17 (AF1924; R\&D Systems), mouse anti-OCT4 (sc-5279; Santa Cruz Biotechnology Inc.), rabbit anti-SOX2 (09-0024; Stemgent), mouse antiSSEA4 (MAB1435; R\&D Systems), goat anti-NANOG (AF1997; R\&D Systems), mouse anti-TRA1-60 (MAB4360; Millipore), rabbit anti-AFP (A000829; DAKO), mouse anti-NKX2.2 and mouse anti-MF20 (DSHB), rabbit anti-TUJ1 (T3952; Sigma-Aldrich), sheep anti-NGN3 (SAB3300089; Sigma-Aldrich), rabbit anti-Ki67 (ab15580, Abcam), rabbit anti-UCN-3 (HPA038281, Sigma-Aldrich), and rabbit anti-ZNT8 (PA5-21010; Thermo Scientific). Appropriate secondary antibodies were obtained from Invitrogen. Quantification of positively stained cells was performed using the Celigo Cytometer system (Cyntellect).

Transplantation. On day 12 of differentiation, cells were dissociated using trypLE (5 minutes at room temperature). Aliquots of 2 to 3 million cells were collected into an eppendorf tube. Cells were spun down, and the supernatant was discarded. 10-15 $\mu$ l Matrigel (BD Biosciences) was added into each tube. Each tube of cell mixture was transplanted under the kidney capsule of an immunodeficient mouse, NOD.Cg-Prkdcsid Il2rg $\mathrm{g}^{\mathrm{t} I \mathrm{~W} \text { Wl }} / \mathrm{SzJ}$ (005557; The Jackson Laboratory) (37), following a previously described protocol (38). For human islet transplantation, approximately 300 human islets obtained from National Disease Research Interchange were transplanted. Three months after transplantation, human C-peptide was determined in the sera of recipient mice. An intraperitoneal glucose tolerance test was performed between 100 and 120 days after transplantation.

Insulin secretion assay. Typically, cells were cultured in 12-well dishes. After 12 days of differentiation, cells were washed for 1 hour in CMRL medium. Cells were then incubated in $300 \mu \mathrm{l} \mathrm{CMRL} \mathrm{medium} \mathrm{containing}$ $5.6 \mathrm{mM}$ glucose for 1 hour, and the medium was collected. Subsequently, $300 \mu \mathrm{l} \mathrm{CMRL}$ medium containing $16.9 \mathrm{mM}$ glucose or other secretagogues was used to treat cells for 1 hour, after which the medium was collected. For in vivo tests, mice were deprived of food overnight with ad libitum access to water. After 12 to 14 hours of fasting, capillary blood glucose concentrations were determined by tail bleed using an Alpha- 
TRACK glucometer (Abbott). Venous blood samples were collected via the submandibular vein. Intraperitoneal glucose was then administered ( $1 \mathrm{mg} / \mathrm{g}$ body weight), and a half hour later blood samples were obtained via the submandibular vein. Blood samples were kept at room temperature for 2 hours, and serum was obtained by centrifuging blood samples at $1,800 \mathrm{~g}$ for 15 minutes.

C-peptide concentrations in media or mouse sera were measure using an ultrasensitive human C-peptide ELISA Kit according to the manufacturer's instructions (Mercodia). All mouse studies were reviewed and approved by the IACUC of Columbia University.

Transmission electron microscopy. Cells were fixed with $2.5 \%$ glutaraldehyde in $0.1 \mathrm{M}$ Sorenson's buffer ( $\mathrm{pH}$ 7.2) for 1 hour. Further processing and imaging of the samples was performed by Diagnostic Service, Department of Pathology and Cell Biology, Columbia University. Insulin granules were defined as electron-dense granular structures using a magnification of $\times 7,500$. The number of insulin granules was determined for 3 cells of each cell line by manual counting.

Statistics. Two-tailed Student's $t$ test was used to determine statistical significance of differences between 2 groups. $P$ values of less than 0.05 were considered significant.

Study approval. Human subject research was reviewed and approved by the Columbia University Stem Cell Committee and the Columbia University IRB. Animal experimentation was approved by the Columbia University IACUC.

\section{Acknowledgments}

We thank Andrew Sproul and Samson Jacob (The New York Stem Cell Foundation) for assistance in Southern blotting. This work was supported by The New York Stem Cell Foundation, The Russell Berrie Foundation, a grant from the Leona M. and Harry B. Helmsley Charitable Trust, The Hunter Eastman Scholar Award in Translational Diabetes Research, The James and Irene Hunter Charitable Fund, an ADA-Mentored Fellowship to H. Hua, and NIH grants RO1 DK52431 and P30DK063608.

Received for publication November 1, 2012, and accepted in revised form April 16, 2013.

Address correspondence to: Rudolph Leibel, 1150 St. Nicholas Avenue, Room 620A, New York, New York 10032, USA. Phone: 212.851.5315; Fax: 212.851.5306; E-mail: rl232@columbia.edu. Or to: Dieter Egli, The New York Stem Cell Foundation, 3960 Broadway, New York, New York 10032, USA. Phone: 212.851.5422; Fax: 212.851.5423; E-mail: d.egli@nyscf.org.

Thomas Ludwig's present address is: Department of Molecular and Cellular Biochemistry, The Ohio State University Wexner Medical Center, Columbus, Ohio, USA.
1. Park IH, et al. Disease-specific induced pluripotent stem cells. Cell. 2008;134(5):877-886.

2. Maehr R, et al. Generation of pluripotent stem cells from patients with type 1 diabetes. Proc Natl Acad Sci US A. 2009;106(37):15768-15773.

3. Kroon E, et al. Pancreatic endoderm derived from human embryonic stem cells generates glucoseresponsive insulin-secreting cells in vivo. Nat Biotechnol. 2008;26(4):443-452.

4. Ohmine S, et al. Reprogrammed keratinocytes from elderly type 2 diabetes patients suppress senescence genes to acquire induced pluripotency. Aging (Albany NY). 2012;4(1):60-73.

5. Teo AK, et al. Derivation of human induced pluripotent stem cells from patients with maturity onset diabetes of the young. J Biol Chem. 2013; 288(8):5353-5356

6. Velho G, et al. Primary pancreatic beta-cell secretory defect caused by mutations in glucokinase gene in kindreds of maturity onset diabetes of the young. Lancet. 1992;340(8817):444-448.

7. Fajans SS, Bell GI, Polonsky KS. Molecular mechanisms and clinical pathophysiology of maturityonset diabetes of the young. N Engl J Med. 2001; 345(13):971-980.

8. Dean L, McEntyre J. The Genetic Landscape of Diabetes. Bethesda, Maryland, USA: National Center for Biotechnology Information; 2004.

9. Ehtisham S, Hattersley AT, Dunger DB, Barrett TG. First UK survey of paediatric type 2 diabetes and MODY. Arch Dis Child. 2004;89(6):526-529.

10. Estalella I, et al. Mutations in GCK and HNF1alpha explain the majority of cases with clinical diagnosis of MODY in Spain. Clin Endocrinol (Oxf). 2007;67(4):538-546.

11. Byrne MM, et al. Insulin secretory abnormalities in subjects with hyperglycemia due to glucokinase mutations. J Clin Invest. 1994;93(3):1120-1130.

12. Njolstad PR, et al. Permanent neonatal diabetes caused by glucokinase deficiency: inborn error of the glucose-insulin signaling pathway. Diabetes. 2003;52(11):2854-2860.

13. Terauchi Y, et al. Pancreatic beta-cell-specific targeted disruption of glucokinase gene. Diabetes mellitus due to defective insulin secretion to glucose. J Biol Chem. 1995;270:30253-30256.
14. Terauchi Y, et al. Glucokinase and IRS-2 are required for compensatory $\beta$ cell hyperplasia in response to high-fat diet-induced insulin resistance. J Clin Invest. 2007;117(1):246-257.

15. Naylor R, Philipson LH. Who should have genetic testing for maturity-onset diabetes of the young? Clin Endocrinol (Oxf). 2011;75(4):422-426.

16. Gidh-Jain M, et al. Glucokinase mutations associated with non-insulin-dependent (type 2) diabetes mellitus have decreased enzymatic activity: implications for structure/function relationships. Proc Natl Acad Sci U S A. 1993;90(5):1932-1936.

17. Fusaki N, Ban H, Nishiyama A, Saeki K, Hasegawa $M$. Efficient induction of transgene-free human pluripotent stem cells using a vector based on Sendai virus, an RNA virus that does not integrate into the host genome. Proc Jpn Acad Ser B Pbys Biol Sci. 2009;85(8):348-362.

18. Zhu H, Lensch MW, Cahan P, Daley GQ. Investigating monogenic and complex diseases with pluripotent stem cells. Nat Rev Genet. 2011;12(4):266-275.

19. D'Amour KA, et al. Production of pancreatic hormone-expressing endocrine cells from human embryonic stem cells. Nat Biotechnol. 2006; 24(11):1392-1401.

20. Redmer T, Diecke S, Grigoryan T, Quiroga-Negreira A, Birchmeier W, Besser D. E-cadherin is crucial for embryonic stem cell pluripotency and can replace OCT4 during somatic cell reprogramming. EMBO Rep. 2011;12(7):720-726.

21. Song WJ, et al. Exendin-4 stimulation of cyclin A2 in beta-cell proliferation. Diabetes. 2008; 57(9):2371-2381.

22. Nostro MC, et al. Stage-specific signaling through TGFbeta family members and WNT regulates patterning and pancreatic specification of human pluripotent stem cells. Development. 2011; 138(5):861-871.

23. Furuya F, Shimura H, Yamashita S, Endo T, Kobayashi T. Liganded thyroid hormone receptoralpha enhances proliferation of pancreatic betacells. J Biol Chem. 2010;285(32):24477-24486.

24. De Krijger RR, Aanstoot HJ, Kranenburg G, Reinhard M, Visser WJ, Bruining GJ. The midgestational human fetal pancreas contains cells coexpressing islet hormones. Dev Biol. 1992;153(2):368-375.
25. Blum B, Hrvatin SS, Schuetz C, Bonal C, Rezania A, Melton DA. Functional beta-cell maturation is marked by an increased glucose threshold and by expression of urocortin 3. Nat Biotechnol. 2012; 30(3):261-264

26. Chimienti F, Favier A, Seve M. ZnT-8, a pancreatic beta-cell-specific zinc transporter. Biometals. 2005;18(4):313-317.

27. Kassem SA, Ariel I, Thornton PS, Scheimberg I, Glaser B. Beta-cell proliferation and apoptosis in the developing normal human pancreas and in hyperinsulinism of infancy. Diabetes. 2000;49(8):1325-1333.

28. Mally MI, Otonkoski T, Lopez AD, Hayek A. Developmental gene expression in the human fetal pancreas. Pediatr Res. 1994;36(4):537-544.

29. Tu J, Tuch BE, Si Z. Expression and regulation of glucokinase in rat islet beta- and alpha-cells during development. Endocrinology. 1999;140(8):3762-3766.

30 . Porat $S$, et al. Control of pancreatic $\beta$ cell regeneration by glucose metabolism. Cell Metab. 2011; 13(4):440-449.

31. Sandhu MS, et al. Common variants in WFS1 confer risk of type 2 diabetes. Nat Genet. 2007;39(8):951-953.

32. Scott LJ, et al. A genome-wide association study of type 2 diabetes in Finns detects multiple susceptibility variants. Science. 2007;316(5829):1341-1345.

33. Dupuis J, et al. New genetic loci implicated in fasting glucose homeostasis and their impact on type 2 diabetes risk. Nat Genet. 2010;42(2):105-116.

34. Vaxillaire $M$, et al. Impact of common type 2 diabetes risk polymorphisms in the DESIR prospective study. Diabetes. 2008;57(1):244-254.

35. Liu GH, et al. Targeted gene correction of laminopathy-associated LMNA mutations in patientspecific iPSCs. Cell Stem Cell. 2011;8(6):688-694.

36. Chen AE, et al. Optimal timing of inner cell mass isolation increases the efficiency of human embryonic stem cell derivation and allows generation of sibling cell lines. Cell Stem Cell. 2009;4(2):103-106.

37. Shultz LD, et al. Human lymphoid and myeloid cell development in NOD/LtSz-scid IL2R gamma null mice engrafted with mobilized human hemopoietic stem cells. J Immunol. 2005;174(10):6477-6489.

38. Szot GL, Koudria P, Bluestone JA. Transplantation of pancreatic islets into the kidney capsule of diabetic mice. JVis Exp. 2007;(9):404. 